\title{
NOUVELLE
}

\section{La première étape de la transmission d'un caractère épigénétique suivie par fluorescence}

Christian Bronner ${ }^{1}$, Marc Mousli², Alain Burger ${ }^{3}$, Yves Mély $^{2}$
${ }^{1}$ Département de génomique fonctionnelle et cancer, Institut de génétique et biologie moléculaire et cellulaire (IGBMC), Université de Strasbourg, CNRS 7104, Inserm U964, 1, rue Laurent Fries, 67404 IIlkirch, France.

${ }^{2}$ Laboratoire de biophotonique et pharmacologie, UMR 7213 CNRS, Université de Strasbourg, faculté de pharmacie, 74, route du Rhin, 67401 Illkirch, France.

${ }^{3}$ Institut de chimie de Nice, Université Côte d'Azur, UMR 7272, CNRS, Parc Valrose, Nice, France. yves.mely@unistra.fr
> Les marques épigénétiques constituent le véritable support de la mémoire cellulaire parce qu'elles permettent à une cellule d'exprimer un nombre bien défini de gènes qui vont déterminer le type cellulaire et son identité $[1,2]$. En effet, pour qu'une cellule du foie soit toujours une cellule hépatique et qu'une cellule du rein soit toujours une cellule rénale, certains gènes doivent demeurer «muets» ou «silencieux» alors que d'autres doivent être constamment exprimés. La capacité à rendre muet un gène est assurée, entre autres, par les cytosines méthylées (une des quatre bases de I'ADN portant un groupement méthyle) positionnées à des endroits stratégiques du génome. La présence de cytosines méthylées empêche l'expression d'un gène et, in fine, la traduction de la protéine correspondante. La méthylation de la cytosine s'effectue sur les 2 brins de l'ADN lorsqu'elle est située à côté d'une guanine. Cette symétrie permet ainsi la transmission semi-conservative de la méthylation de I'ADN (Figure 1) selon un modèle qui fait appel à des acteurs bien particuliers (voir ci-après).
Le défi, pour une cellule qui prolifère, est de recopier fidèlement les profils de méthylation à l'emplacement exact sur le génome, et de les transmettre à la descendance afin de préserver l'identité cellulaire. Une altération de cette fidélité signifierait, pour la cellule, une perte des capacités à assurer ses fonctions et, dans certains cas, l'apparition d'anomalies pouvant conduire à des cancers ou des maladies neuro-dégénératives de type maladie d'Alzheimer [3].

La cytosine méthylée n'existe pas, comme telle, à l'état naturel et c'est donc une

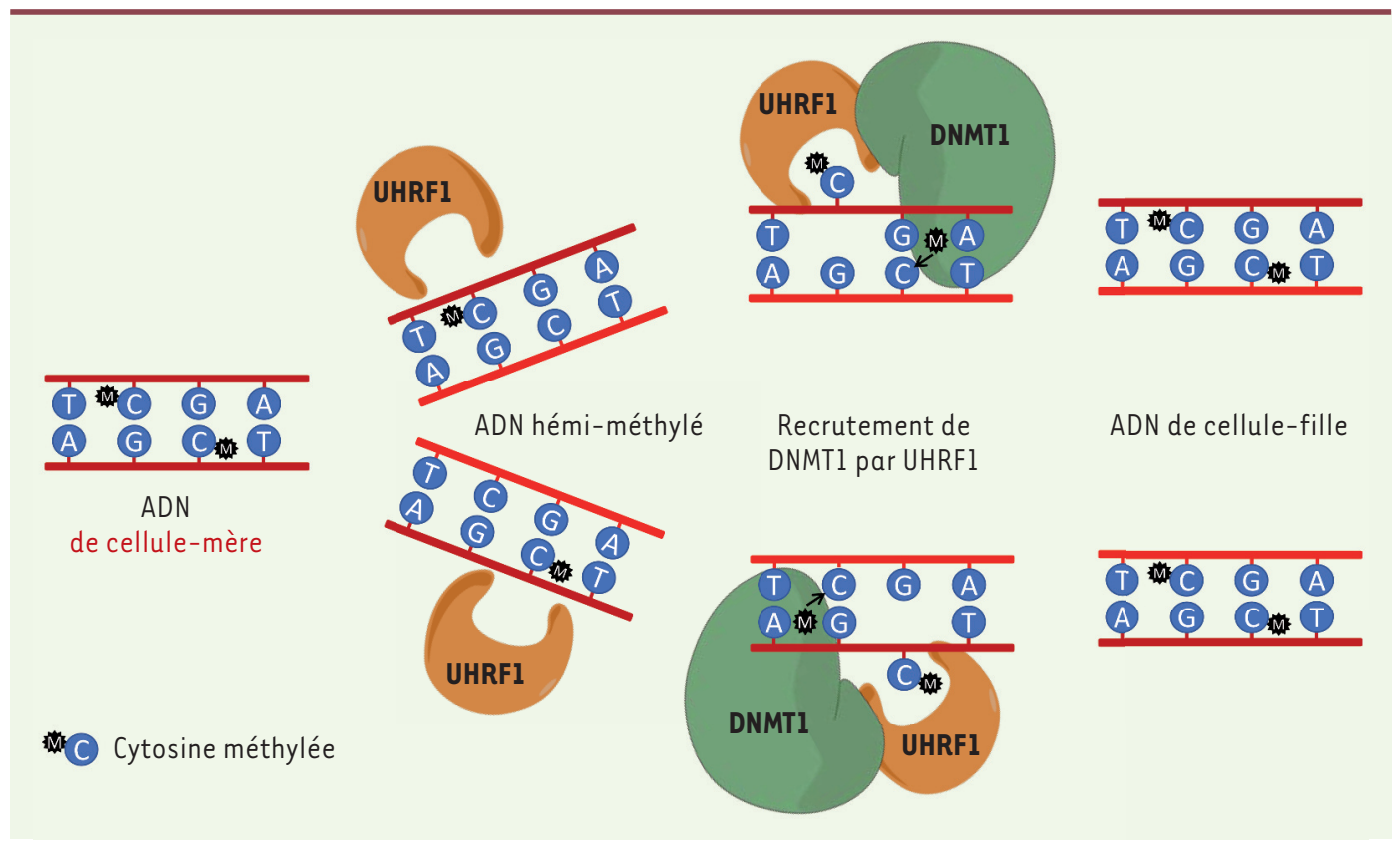

Figure 1. Représentation schématique de la coopération entre UHRF1 et DNMT1 pour la réplication de la méthylation de l'ADN. Dans la cellule-mère, I'ADN est méthylé sur ses deux brins au niveau de sites CpG. Lors de la duplication de I'ADN, il y a synthèse d'un nouveau brin dont les cytosines ne sont pas méthylées. L'ADN généré est donc un ADN hémi-méthylé (un seul des 2 brins est méthylé). Les sites CpG hémi-méthylés sont reconnus par la protéine UHRFl, grâce à son domaine SRA (set and ring associated). Cette reconnaissance est suivie d'un basculement de la cytosine méthylée hors de la double hélice d'ADN, afin de permettre à UHRFI de recruter la protéine DNMTI dans le but de lui indiquer quelle cytosine est à méthyler sur l'autre brin. DNMTl : ADN méthyltransférase 1 ; UHRFl : ubiquitin-like containing PHD and RING finger domains 1 . 


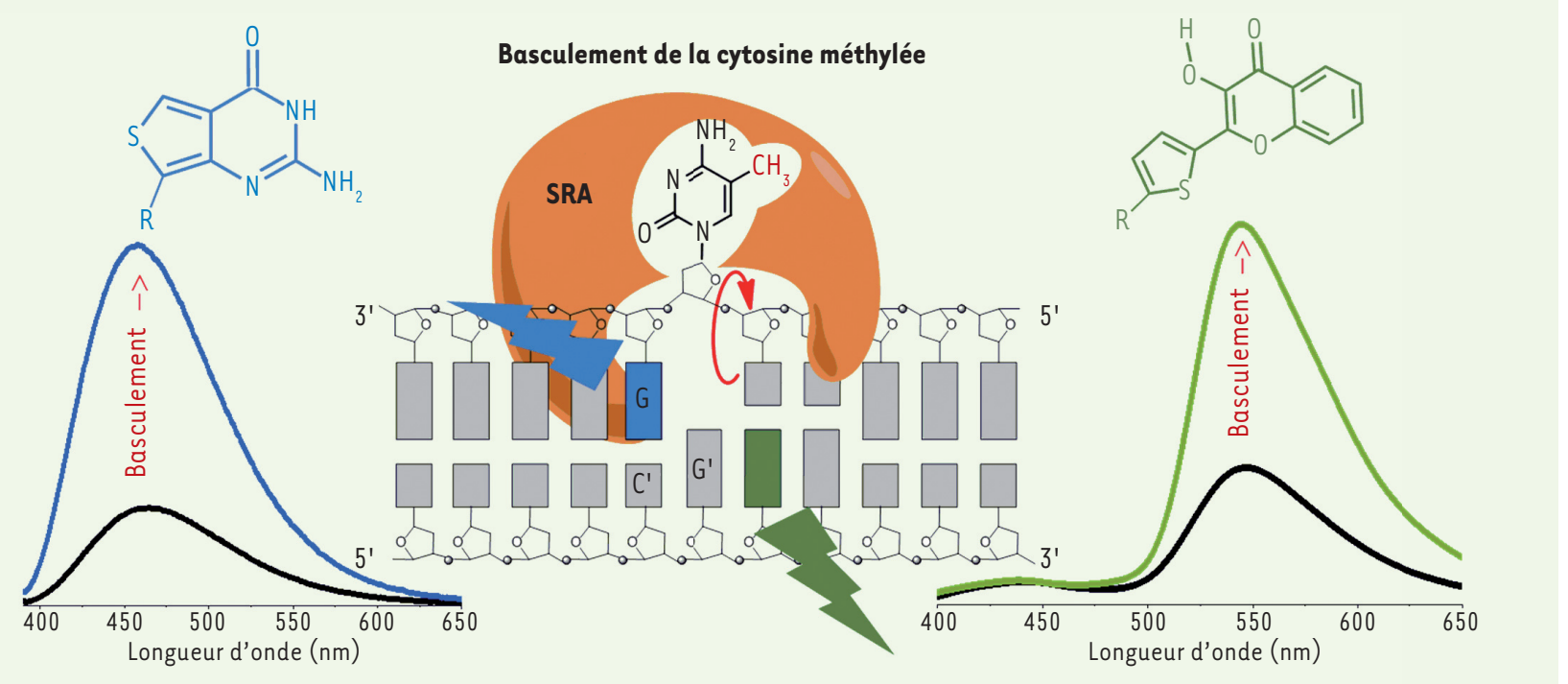

Figure 2. Suivi par fluorescence du basculement de la cytosine méthylée par le domaine SRA (set and ring associated) de la protéine UHRF1. L’ADN est marqué par un analogue nucléosidique fluorescent : la thiénoguanosine (thG, représentée en bleu) ou la 2-thiényl-3-hydroxychromone (3HCnt, représentée en vert). Au niveau du site $\mathrm{mCpG}$, le domaine SRA fait basculer la cytosine méthylée, ce qui va stabiliser la liaison de ce domaine à I'ADN hémi-méthylé et permettre le recrutement de la protéine DNMTl. Le basculement de la cytosine méthylée à l'extérieur de I'hélice d'ADN se traduit par une forte augmentation de la fluorescence de la thG ou de la 3HCnt (figure adaptée de l'article de Kilin et al. [6]). DNMTl : ADN méthyltransférase 1 ; UHRFl : ubiquitin-like containing PHD and RING finger domains 1.

cytosine nue qui est incorporée lors de la duplication de l'ADN, provoquant ainsi l'apparition d'un ADN hémi-méthylé, où un seul des deux brins de l'ADN est méthylé. L'une des premières étapes de la duplication du profil de méthylation implique, par conséquent, la reconnaissance de l'ADN hémi-méthylé. La méthylation de l'autre brin de l'ADN est assurée par la protéine DNMTI (ADN méthyltransférase 1) [4] $(\rightarrow)$.

Pour aider cette

$(\rightarrow)$ Voir la Nouvelle de $M$. Weber, $m / s n^{\circ} 5$, mai 2011, page 483

dernière à identifier les cytosines à méthyler, il lui faut un guide, en l'occurrence la protéine UHRFl (ubiquitinlike, containing PHD and RING finger domains 1) dont le domaine SRA (set and ring associated) reconnaît I'ADN hémi-méthylé [5]. Cette reconnaissance est suivie d'un basculement de la cytosine méthylée, hors de la double hélice de I'ADN, afin de permettre à l'UHRFl de marquer une pause, probablement pour «dialoguer» avec l'enzyme DNMTl afin de lui indiquer quelle cytosine est à méthyler sur l'autre brin (Figure 1).
La manière dont s'opère le dialogue de ce ménage à trois, entre l'UHRFl, la DNMTl et l'ADN hémi-méthylé, reste un mystère. Sa compréhension ouvrirait des perspectives intéressantes pour corriger les erreurs ou inhiber le processus d'hyperméthylation. Prenons l'exemple du cancer, caractérisé notamment par la mise en «silence» de gènes bénéfiques comme les gènes suppresseurs de tumeurs. Les promoteurs de ces gènes sont fréquemment hyperméthylés car de nombreuses cytosines sont méthylées en amont du site d'initiation de la transcription. Lorsqu'ils sont déméthylés ou lorsque les profils de méthylation ne sont pas dupliqués, ces gènes peuvent à nouveau s'exprimer, avec pour conséquence l'activation de l'apoptose, un processus conduisant à la mort de la cellule. Ainsi, cibler la transmission des profils de méthylation serait un moyen ingénieux d'inciter la cellule cancéreuse à se «suicider» en lui permettant de ré-exprimer les gènes suppresseurs de tumeurs.
Dans ce contexte, l'objectif de notre travail publié dans la revue J Am Chem Soc, a été de suivre et de caractériser la dynamique du basculement des cytosines méthylées sous l'effet du domaine SRA de la protéine UHRFl qui reconnaît I'ADN hémi-méthylé [6]. Pour atteindre cet objectif, nous avons utilisé deux analogues fluorescents de nucléosides très innovants, la 2-thiényl-3-hydroxychromone (3HCnt) capable de substituer toutes les nucléobases de I'ADN [7] et la thiénoguanosine (thG) qui remplace parfaitement la guanine [8, 9]. En incorporant la $3 \mathrm{HCnt}$ ou la thG à des positions choisies d'un ADN double brin hémi-méthylé ou non méthylé, nous avons montré par spectroscopie de fluorescence qu'il était possible de suivre de manière sensible et fidèle le basculement des cytosines méthylées, induit par le domaine SRA de la protéine UHRFI (Figure 2). En effet, le domaine SRA induit une forte augmentation de la fluorescence de ces deux sondes, indiquant la très grande sensibilité de ces analogues fluorescents de nucléosides 
aux changements structuraux mineurs accompagnant le basculement des bases [10]. Cette forte augmentation ne se produit que pour les séquences hémiméthylées et non pour les séquences non méthylées correspondantes. En outre, cette forte augmentation de fluorescence est abolie quand le domaine SRA est remplacé par une protéine mutante incapable d'induire un basculement de base [10]. Par ailleurs, nous avons vérifié que (1) les analogues thG et $3 \mathrm{HCnt}$ n'affectent pas ou peu la stabilité du double brin d'ADN dans lequel ils sont insérés, et (2) qu'aux positions où ces sondes sont sensibles au basculement de base induit par SRA, ce domaine conserve une affinité préférentielle pour les séquences hémi-méthylées par rapport aux séquences non méthylées. Ces sondes ont permis également de déterminer par une technique de «stoppedflow $^{1} \gg$ que la liaison du domaine SRA avec I'ADN est rapide et donc compatible avec l'activité de lecture de I'UHRFl pour trouver les sites à méthyler sur le brin d’ADN néo-synthétisé [6, 11]. En revanche, l'interaction de SRA avec la cytosine méthylée et le bascu-

${ }^{1}$ Analyse de cinétique rapide d'interactions moléculaires en flux arrêté. lement de cette dernière dans la poche de liaison constituent un événement lent, d'une durée compatible avec le recrutement de la DNMTl par I'UHRFl à l'emplacement adéquat. À nouveau, cette cinétique lente disparaît quand le domaine SRA est remplacé par un motif mutant incapable d'induire un basculement de base. Nos résultats sont, par conséquent, en parfaite cohérence avec l'image de l'UHRFl servant de guide à la méthyl-transférase DNMTl pour identifier les cytosines à méthyler.

Cette étude donne un nouvel éclairage sur le fonctionnement du tandem UHRFI/DNMTl et illustre la complexité du mécanisme de transmission d'un caractère épigénétique. L'étape suivante est de comprendre comment UHRFl dialogue avec la DNMTl pour lui indiquer quelle est la cytosine à cibler afin de boucler le processus de duplication des profils de méthylation. La technologie mise en œuvre devrait également permettre d'envisager le développement de tests de criblage pour identifier des inhibiteurs du basculement de la cytosine méthylée. $\diamond$

The first stage of transmission of an epigenetic character followed by fluorescence

\section{LIENS D'INTÉRÊT}

Les auteurs déclarent n'avoir aucun lien d'intérêt concernant les données publiées dans cet article.

\section{RéFÉRENCES}

1. Allis $C D$, Jenuwein $T$. The molecular hallmarks of epigenetic control. Nat Rev Genet 2016; 17 : 487-500.

2. Jeltsch $A$, Jurkowska RZ. New concepts in DNA methylation. Trends Biochem Sci 2014 ; 39 : 310-8.

3. Heyn $\mathrm{H}$, Esteller M. DNA methylation profiling in the clinic: applications and challenges. Nat Rev Genet $2012 ; 13: 679-92$.

4. Weber, M. La méthylation de l'ADN, un acteur-clé de la pluripotence. Med Sci (Paris) 2011 ; 27 : 483-5.

5. Bronner C, Krifa M, Mousli M. Increasing role of UHRFl in the reading and inheritance of the epigenetic code as well as in tumorogenesis. Biochem Pharmacol 2013 ; 86 : 1643-9.

6. Kilin V, Gavvala K, Barthes NP, et al. Dynamics of methylated cytosine flipping by UHRFI. J Am Chem Soc 2017 ; 139 : 2520-8

7. Dziuba D, Postupalenko Vy, Spadafora M, et al. A universal nucleoside with strong two-band switchable fluorescence and sensitivity to the environment for investigating DNA interactions. J Am Chem Soc 2012 ; 134 : 10209-13.

8. Shin D, Sinkeldam RW, Tor Y. Emissive RNA alphabet. J Am Chem Soc 2011 ; 133 : 14912-5.

9. Sholokh M, Sharma R, Shin D, et al. Conquering 2-aminopurine's deficiencies: highly emissive isomorphic guanosine surrogate faithfully monitors guanosine conformation and dynamics in DNA. J Am Chem Soc 2015 ; 137 : 3185-8.

10. Avvakumov GV, Walker JR, Xue S, et al. Structural basis for recognition of hemi-methylated DNA by the SRA domain of human UHRFI. Nature 2008 ; $455: 822-5$.

11. Greiner VJ, Kovalenko L, Humbert N, et al. Siteselective monitoring of the Interaction of the SRA domain of UHRFl with target DNA sequences labeled with 2-aminopurine. Biochemistry 2015; $54: 6012-20$.

\title{
Filière nationale de santé neuromusculaire Filnemus
}

\author{
$4^{e}$ Journée annuelle \\ Mardi 7 novembre 2017 \\ $9 \mathrm{~h} 30$ \\ Institut Imagine \\ Hôpital Necker-Enfants Malades - Paris - France
}

Y seront notamment abordées les actualités de la Filière elle-même et du $3^{\text {e }}$ Plan National Maladies Rares, ainsi que les travaux des différentes commissions de Filnemus.

Renseignements et inscriptions www.filnemus.fr

FILNEMUS est une des 23 Filières de Santé Maladies Rares (FSMR) retenues par le Ministère dans le cadre du second plan national maladies rares 2011-2016. Les affections relevant de la filière FILNEMUS incluent les maladies du muscle (myopathies), les maladies de la jonction neuromusculaire, les maladies rares du nerf périphérique et les amyotrophies spinales infantiles. À ce jour, on compte, en France, entre 40000 et 50000 personnes atteintes de pathologie neuromusculaire. 